\title{
Nuclear Medicine Methods in the Diagnosis of Bone Metastases
}

\author{
(1) Yasemin ŞANLI \\ Department of Nuclear Medicine, Istanbul University, Istanbul Faculty of Medicine, İstanbul-Turkey
}

\section{Introduction}

Bone is a frequent site of metastases in advancedstage cancer and approximately half of the new cancer patients can metastasize to the bone.[1] In adult metastatic patients, bone metastases rank third after lung and liver metastases. [2] Post-mortem studies have shown that bone metastases are present in approximately $70 \%$ of the patients with breast and prostate cancer and $20-30 \%$ of those with lung and gastrointestinal cancer.[3] In proportion to the increased prevalence of bone metastases, the patients experience pain, pathological fractures, restricted mobility, hypercalcemia, spinal cord or nerve root entrapment, and an increase in the symptoms negatively affecting the quality of life. [4] While the detection of metastases is a prognostic factor, early diagnosis and optimal treatment improve the overall survival rate and quality of life in several tumors.[5] There is some evidence showing that patients who have predominantly bone metastatic disease have better survival and favorable prognosis than those with visceral and oligometastatic diseases. [6] Bone metastases might be osteolytic, osteosclerotic, or more frequently, mixed. They are most frequently localized in the vertebrae, costae, and the metaphyseal region of the long bones, which are rich in red bone marrow.[7] In the vertebrae, metastases are most frequently localized in the lumbar (52\%), followed by thoracic (36\%), and rarely cervical (12\%).[8]

Disease-specific imaging agents are updated with the introduction of novel radiopharmaceuticals in $\mathrm{Nu}$ clear Medicine. Herein, we present the scintigraphy and positron emission tomography (PET) imaging agents used for the imaging of bone metastases in routine clinical practice.

\section{Bone Scintigraphy}

Bone scintigraphy, which allows imaging of the complete body, is the oldest and the most frequently used imaging modality among the scintigraphy methods with its low cost, easy accessibility, adequate physical and effective half-life. Technetium-99m (99mTc)-labeled methylene diphosphonate (MDP) is the most frequently used bone scintigraphy agent. DP compounds are localized in the osseous matrix through binding to hydroxyapatite crystals by chemical absorption. Approximately $50 \%$ of the radiopharmaceuticals are localized within the bones, while the remaining are excreted through kidneys. Peak bone uptake occurs approximately 1 hour after intravenous injection. The greatest target-background ratio occurs after 6-12 h. Imaging is performed between 2 to $4 \mathrm{~h}$ after the injection due to background clearance and a 6-h half-life of 99mTc. Although 30-70\% demineralization is necessary to visualize bone metastases in radiography, bone scintigraphy allows visualization of the metastases 2-18 months before they are visible on direct radiography.[1]

Bone scintigraphy has $78 \%$ sensitivity in the detection of bone metastases; however, its specificity rates are lower (48\%) due to high false positivity rates.[9] Additional imaging techniques such as radiography, CT, or MRI might be necessary for differential diagnosis of metastases, particularly due to osteodegenerative changes. Nevertheless, the low spatial resolution of scintigraphy (approximately $1 \mathrm{~cm}$ ) may lead to falsepositive results in the detection of metastases.[10] Moreover, false-negative results may also be obtained in osteolytic lesions with a limited reactive osteoblastic reaction, such as renal cell carcinoma metastases since the activity accumulation is typically low.[11] When 
bone metastases are large and diffuse, bone scintigraphy might appear usual due to the aggregating nature of the lesions. This is called "super scan" imaging. In the latter, diffuse activity increase is observed in the axial skeletal system, and it should also be considered when the kidneys, which are expected to be observed physiologically due to renal elimination, are observed with no or very low enhancement.[11]

Three-dimensional images in addition to planar bone scintigraphy, allow the single-photon emission computerized tomography (SPECT) imaging of the skeletal system. The same radionuclide (99mTc-MDP) is used for the imaging; after planar whole-body imaging is performed, sectional three-dimensional images are recorded with the camera rotating $360^{\circ}$ around the patient in certain areas limited by the camera head. SPECT is an imaging method using the emission technique, based on the activity formation, which already exists in the patient before the planar imaging, with no additional radiation dose given. SPECT images allow the visualization of three-dimensional sections, thereby better localization of the abnormal radionuclide uptake. The sensitivity and specificity of the SPECT in the detection of bone metastases are $87 \%$ and $91 \%$, respectively.[9] Moreover, anatomical details are obtained for the determination of the localization of suspected bone lesions using hybrid devices equipped with CT components (SPECT/CT). Thereby, the low specificity of the planar imaging can be increased substantially. A recent meta-analysis demonstrated that the sensitivity and specificity rates increase to $93 \%$ and $96 \%$, respectively, with the addition of SPECT/CT to imaging in the detection of bone metastases.[12] In another metaanalysis comparing the diagnostic accuracy of the $\mathrm{CT}$, SPECT, and MRG imaging, the sensitivity rates were shown to be $77.1 \%$ (95\% confidence interval [CI] 0.730.81 ), $76.8 \%$ (95\% CI $0.73-0.81$ ), and $90.4 \%$ (95\% CI 0.87-0.93), respectively, while the specificity rates were 83.2\% (95\% CI $0.80-0.86$ ), $96.3 \%$ (95\% CI $0.95-0.97$ ), and $96.0 \%$ (95\% CI 0.95-0.97), respectively.[13]

\section{PET imaging agents}

PET imaging can be performed using different radiopharmaceuticals chosen, based on the primary disease.

${ }^{18} \mathrm{~F}-\mathrm{NaF}$ PET: Sodium fluoride $(\mathrm{NaF})$ is an osteotropic compound. It has a similar uptake mechanism and higher first-pass extraction than 99mTcMDP used in PET imaging.[14] Regional extraction of ${ }^{18} \mathrm{~F}-\mathrm{NaF}$ from plasma to bone is 10 -fold higher in metastases compared to normal bone tissue.[15] Its major limita- tions include cyclotron requirement for the production of ${ }^{18} \mathrm{~F}$ and higher cost than the bone scintigraphy. Imaging performed 1-h after the injection is one of the advantages over bone scintigraphy. In their meta-analysis comparing bone SPECT and ${ }^{18} \mathrm{~F}-\mathrm{NaF}$ PET/CT imaging, Sheikhbahaei et al.[16] showed that the sensitivity and specificity are $90 \%$ (95\% CI $0.86-0.93)$ and $85 \%$ (95\% CI 0.80-0.90) for SPECT and 97\% (95\% CI 0.95-0.98) and $84 \%$ (95\% CI $0.80-0.90)$ for ${ }^{18} \mathrm{~F}-\mathrm{NaF} \mathrm{PET} / \mathrm{BT}$, respectively. These results indicate that the contribution of ${ }^{18} \mathrm{~F}-\mathrm{NaF}$ PET/CT to SPECT imaging is to increase its sensitivity in the detection of metastases.

${ }^{18} \mathrm{~F}$-Fluorodeoxyglucose $\left({ }^{18} \mathrm{~F}-\mathrm{FDG}\right):{ }^{18} \mathrm{~F}-\mathrm{FDG}$ PET is functional imaging that shows the cellular biodistribution of glucose metabolism. It is a widely used radiopharmaceutical in oncology due to the greatly increased glucose metabolism of the tumor cells. While the osteoblastic response to bone destruction by tumor cells and accompanying blood flow increase is observed in the bone scintigraphy, ${ }^{18} \mathrm{~F}-\mathrm{FDG}$ PET imaging has a higher success rate in showing lytic bone metastases.[17] The sensitivity of ${ }^{18} \mathrm{~F}-\mathrm{FDG}$ PET/CT may vary by different histopathologies. For example, due to decreased metabolic activity in sclerotic bone metastases, bone scintigraphy has a higher sensitivity than FDG PET/CT.[11] Moreover, its other limitations include the false positivity in infections and inflammation due to the accumulation of ${ }^{18} \mathrm{~F}$-FDG PET in metabolically active tissues. FDG PET/CT shows the bone metastases with a sensitivity of $98 \%$ and a specificity of $56 \%$.[9]

${ }^{68}$ Gaprostate-specific membrane antigen (PSMA): PET imaging using PSMA in patients with prostate cancer is greatly promising in the staging of the disease and recurrence assessment. Caglar et al.[18] examined the contribution of this imaging modality to bone scintigraphy. They showed that PSMA images were found to be superior to bone scintigraphy in the assessment of metastatic disease in spite of it has a higher cost than bone scintigraphy. Moreover, it has been stated that bone scintigraphy can detect bone metastases with a sensitivity of $75 \%$, and this rate increase to $98.2 \%$ using ${ }^{68} \mathrm{Ga}$-PSMA PET/CT. In a study comparing ${ }^{18} \mathrm{~F}-\mathrm{FDG}$ $\mathrm{PET} / \mathrm{CT}$ and bone scintigraphy in the assessment of bone metastases in patients diagnosed with high-grade prostate cancer, it has been stated that ${ }^{18} \mathrm{~F}-\mathrm{FDG}$ PET/ $\mathrm{CT}$ shows the lesions with higher sensitivity and accuracy (100\% vs. $78.8 \%$; $98.7 \%$ vs. $98.2 \%)$.[19] In a metaanalysis comparing the diagnostic performance of ${ }^{68} \mathrm{Ga}-$ PSMA PET/CT, choline PET/CT, and ${ }^{18} \mathrm{~F}-\mathrm{NaF}$ PET/CT, MRI and bone scintigraphy, patient-based (the per-patient pooled sensitivity) sensitivity rates of the imaging modalities were found to be $0.97,0.87,0.96,0.91$, and 
0.86 , respectively and the specificity rates to be 1.00 , 0.99, 0.97, 0.96, and 0.95, respectively.[20] According to the meta-analysis results, the highest patient-based sensitivity and specificity rates in the detection of bone metastases were determined by ${ }^{68} \mathrm{Ga}$-PSMA PET/CT imaging in prostate cancer. The sensitivity of ${ }^{18} \mathrm{~F}-\mathrm{NaF}$ PET/CT and MR was higher than Choline PET/CT and bone scintigraphy. The specificity of PSMA PET/CT was found to be significantly higher than bone scintigraphy. It has been accepted as a preferred agent for the evaluation of prostate cancer bone metastases and all metastatic foci, especially in the high-risk patient group.

\section{References}

1. Davila D, Antoniou A, Chaudhry MA. Evaluation of osseous metastasis in bone scintigraphy. Semin Nucl Med 2015;45(1):3-15.

2. Even-Sapir E. Imaging of malignant bone involvement by morphologic, scintigraphic, and hybrid modalities. J Nucl Med 2005;46(8):1356-67.

3. Coleman RE, Rubens RD. The clinical course of bone metastases from breast cancer. Br J Cancer 1987;55(1):61-6.

4. Kubota H, Soejima T, Sulaiman NS, Sekii S, Matsumoto Y, Ota Y, et al. Predicting the survival of patients with bone metastases treated with radiation therapy: a validation study of the Katagiri scoring system. Radiat Oncol 2019;14(1):13.

5. Lutz S, Berk L, Chang E, Chow E, Hahn C, Hoskin P, et al. Palliative radiotherapy for bone metastases: an ASTRO evidence-based guideline. Int J Radiat Oncol Biol Phys 2011;79(4):965-76.

6. Cook GJR, Goh V. Molecular imaging of bone metastases and their response to therapy. J Nucl Med 2020;61(6):799-806.

7. Bussard KM, Gay CV, Mastro AM. The bone microenvironment in metastasis; what is special about bone? Cancer Metastasis Rev 2008;27(1):41-55.

8. Talbot JN, Paycha F, Balogova S. Diagnosis of bone metastasis: recent comparative studies of imaging modalities. Q J Nucl Med Mol Imaging 2011;55(4):374410.

9. O'Sullivan GJ, Carty FL, Cronin CG. Imaging of bone metastasis: An update. World J Radiol 2015;7(8):20211.

10. Cuccurullo V, Cascini GL, Tamburrini O, Rotondo A, Mansi L. Bone metastases radiopharmaceuticals: an overview. Curr Radiopharm 2013;6(1):41-7.
11. Bäuerle T, Semmler W. Imaging response to systemic therapy for bone metastases. Eur Radiol 2009;19(10):2495-507.

12. Mohd Rohani MF, Zanial AZ, Suppiah S, Phay Phay K, Mohamed Aslum Khan F, Mohamad Najib FH, et al. Bone single-photon emission computed tomography/computed tomography in cancer care in the past decade: a systematic review and meta-analysis as well as recommendations for further work. Nucl Med Commun 2021;42(1):9-20.

13. Yang HL, Liu T, Wang XM, Xu Y, Deng SM. Diagnosis of bone metastases: a meta-analysis comparing ${ }^{18} \mathrm{FDG}$ PET, CT, MRI and bone scintigraphy. Eur Radiol 2011;21(12):2604-17.

14. Langsteger W, Rezaee A, Pirich C, Beheshti M. (18)FNaF-PET/CT and (99 m)Tc-MDP bone scintigraphy in the detection of bone metastases in prostate cancer. Semin Nucl Med 2016;46(6):491-501.

15. Bastawrous S, Bhargava P, Behnia F, Djang DS, Haseley DR. Newer PET application with an old tracer: role of $18 \mathrm{~F}-\mathrm{NaF}$ skeletal PET/CT in oncologic practice. Radiographics 2014;34(5):1295-316.

16. Sheikhbahaei S, Jones KM, Werner RA, SalasFragomeni RA, Marcus CV, Higuchi T, et al. (18)FNaF-PET/CT for the detection of bone metastasis in prostate cancer: a meta-analysis of diagnostic accuracy studies. Ann Nucl Med 2019;33(5):351-61.

17. Deeks JJ. Systematic reviews in health care: Systematic reviews of evaluations of diagnostic and screening tests. BMJ 2001;323(7305):157-62.

18. Caglar M, Tuncel M, Yildiz E, Karabulut E. Bone scintigraphy as a gatekeeper for the detection of bone metastases in patients with prostate cancer: comparison with Ga-68 PSMA PET/CT. Ann Nucl Med 2020;34(12):932-41.

19. Otis-Chapados S, Goulet CR, Dubois G, Lavallée É, Dujardin T, Fradet Y, et al. (18)F-Fluorodeoxyglucose positron emission tomography/computed tomography (PET/CT) is accurate for high-grade prostate cancer bone staging when compared to bone scintigraphy. Can Urol Assoc J 2021 Mar 18. doi: 10.5489/cuaj.7107. Online ahead of print.

20. Zhou J, Gou Z, Wu R, Yuan Y, Yu G, Zhao Y. Comparison of PSMA-PET/CT, choline-PET/CT, NaF-PET/ $\mathrm{CT}, \mathrm{MRI}$, and bone scintigraphy in the diagnosis of bone metastases in patients with prostate cancer: a systematic review and meta-analysis. Skeletal Radiol 2019;48(12):1915-24. 